\title{
Attenuated Live Listeria monocytogenes Encoding KRAS G12D
}

National Cancer Institute

\section{Source}

National Cancer Institute. Attenuated Live Listeria monocytogenes Encoding KRAS G12D. NCI Thesaurus. Code C158466.

An off-the-shelf, plasmid DNA-based cancer vaccine composed of a live-attenuated strain of the Gram-positive bacterium Listeria monocytogenes (Lm) carrying a plasmid vector encoding multiple, not yet disclosed, tumor associated antigens (TAAs) and sequence peptides associated with commonly occurring hotspot mutations, including the aspartic acid substitution for glycine at position 12 (G12D) in KRAS, with potential immunostimulatory and antineoplastic activities. Upon administration, ADXS-503 is taken up by antigen presenting cells (APCS) and the TAAs are processed and presented to immune cells by both major histocompatibility complex (MHC) I and II molecules. This leads to an increase in antigen-specific CD8-positive T-cells and gamma/delta T-cells within the tumor microenvironment (TME) and an inhibition of immunosuppressive tumor-infiltrating T-regulatory cells (Tregs) and myeloid-derived suppressor cells (MDSCs). This may lead to tumor cell death in cells expressing TAAs that are shared across multiple tumor types. The KRAS G12D mutation is thought to drive tumorigenesis and progression in some cancers. 